\title{
COFINITELY RADICAL SUPPLEMENTED AND COFINITELY WEAK RADICAL SUPPLEMENTED LATTICES
}

\author{
CELIL NEBIYEV AND HASAN HÜSEYIN ÖKTEN
}

Received 04 February, 2020

\begin{abstract}
In this work, cofinitely radical supplemented and cofinitely weak radical supplemented lattices are defined and some properties of them are investigated. Let $L$ be a lattice, $I$ be a nonempty index set and $a_{i} \in L$ for every $i \in I$. If $1=\underset{i \in I}{\vee} a_{i}$ and $a_{i} / 0$ is cofinitely (weak) radical supplemented for every $i \in I$, then $L$ is also cofinitely (weak) radical supplemented. Let $L$ be a cofinitely (weak) radical supplemented lattice and $a \in L$. Then $1 / a$ is also cofinitely (weak) radical supplemented. Let $L$ be a lattice. Then $L$ is cofinitely weak radical supplemented if and only if every cofinite element of $1 / r(L)$ is a direct summand of $1 / r(L)$.
\end{abstract}

2010 Mathematics Subject Classification: 06C05; 06C15

Keywords: lattices, small elements, supplemented lattices, generalized (radical) supplemented lattices

\section{INTRODUCTION}

Throughout this paper, all lattices are complete modular lattices with the smallest element 0 and the greatest element 1 . Let $L$ be a lattice, $a, b \in L$ and $a \leq b$. A sublattice $\{x \in L \mid a \leq x \leq b\}$ is called a quotient sublattice, denoted by $b / a$. An element $a^{\prime}$ of a lattice $L$ is called a complement of $a$ if $a \wedge a^{\prime}=0$ and $a \vee a^{\prime}=1$ (in this case $a$ and $a^{\prime}$ are said to be direct summands of $L$ and denoted by $1=a \oplus a^{\prime}$ ). A lattice $L$ is said to be complemented if each element of $L$ has at least one complement in $L$. An element $c$ of $L$ is said to be compact if for every subset $X$ of $L$ such that $c \leq \vee X$ there exists a finite $F \subset X$ such that $c \leq \vee F$. A lattice $L$ is said to be compactly generated if each of its elements is a join of compact elements. A lattice $L$ is said to be compact if 1 is a compact element of $L$. An element $a$ of a lattice $L$ is said to be cofinite if $1 / a$ is compact. An element $a$ of $L$ is said to be small or superfluous if $a \vee b \neq 1$ holds for every $b \neq 1$ and denoted by $a \ll L$. The meet of all the maximal $(\neq 1)$ elements of a lattice $L$ is called the radical of $L$ and denoted by $r(L)$. An element $c$ of $L$ is called a supplement of $b$ in $L$ if it is minimal for $b \vee c=1$. $a$ is a supplement of $b$ in a lattice $L$ if and only if $a \vee b=1$ and $a \wedge b \ll a / 0$. $L$ is called a supplemented lattice if every element of $L$ has a supplement in $L$. We say that an element $b$ of $L$ lies above an element $a$ of $L$ if $a \leq b$ and $b \ll 1 / a$. $L$ is said to be hollow if every element $(\neq 1)$ 
is superfluous in $L$ and $L$ is said to be local if $L$ has the greatest element $(\neq 1)$. An element $a$ of $L$ is called a weak supplement of $b$ in $L$ if $a \vee b=1$ and $a \wedge b \ll L$. $L$ is called a weakly supplemented lattice, if every element of $L$ has a weak supplement in $L$. It is clear that every supplemented lattice is weakly supplemented. An element $a$ of $L$ is called a generalized (radical) supplement (or briefly, Rad-supplement) of $b$ in $L$ if $a \vee b=1$ and $a \wedge b \leq r(a / 0) . L$ is said o be radical (generalized) supplemented if every element of $L$ has a Rad-supplement in $L$.

More information about supplemented lattices are in [1,2] and [5]. More results about supplemented modules are in [9] and [10]. The definitions of generalized supplemented modules and some properties of them are in [8]. More information about cofinitely Rad-supplemented modules are in [7]. We generalize cofinitely Radsupplemented modules to lattices.

\section{Cofinitely Radical Supplemented Lattices}

In this part, cofinitely radical supplemented lattices are defined and some properties of them are given.

Definition 1. Let $L$ be a lattice. If every cofinite element of $L$ has a Rad-supplement in $L$, then $L$ is called a cofinitely radical supplemented (or cofinitely Rad-supplemented) lattice.

Clearly we can see that every cofinitely supplemented lattice is cofinitely Radsupplemented. Hollow and local lattices are cofinitely Rad-supplemented.

Proposition 1. Let $L$ be a compact lattice. Then $L$ is cofinitely Rad-supplemented if and only if $L$ is Rad-supplemented.

Proof. Clear, since every element of $L$ is cofinite.

Lemma 1. Let $L$ be a lattice, $a \in L$ and $x$ be a cofinite element of $L$. If $x \vee a$ has a Rad-supplement in L and a/0 cofinitely Rad-supplemented, then $x$ has a Radsupplement in $L$.

Proof. Let $b$ be a Rad-supplement of $x \vee a$ in $L$. Then $x \vee a \vee b=1$ and $(x \vee a) \wedge b \leq$ $r(b / 0)$. Since $x$ is a cofinite element of $L$, we clearly see that $x \vee b$ is a cofinite element of $L$. Then by $\frac{1}{x \vee b}=\frac{x \vee a \vee b}{x \vee b} \cong \frac{a}{a \wedge(x \vee b)}, a \wedge(x \vee b)$ is a cofinite element of $a / 0$. Since $a / 0$ is cofinitely Rad-supplemented, $a \wedge(x \vee b)$ has a Rad-supplement $c$ in $a / 0$. Here $(a \wedge(x \vee b)) \vee c=a$ and $c \wedge(x \vee b)=c \wedge a \wedge(x \vee b) \leq r(c / 0)$. Hence $1=x \vee a \vee b=x \vee b \vee(a \wedge(x \vee b)) \vee c=x \vee b \vee c$ and $x \wedge(b \vee c) \leq(b \wedge(x \vee c)) \vee$ $(c \wedge(x \vee b)) \leq(b \wedge(x \vee a)) \vee r(c / 0) \leq r(b / 0) \vee r(c / 0) \leq r((b \vee c) / 0)$. Thus $b \vee c$ is a Rad-supplement of $x$ in $L$.

Corollary 1. Let $L$ be a lattice, $a_{1}, a_{2}, \ldots, a_{n} \in L$ and $x$ be a cofinite element of L. If $x \vee a_{1} \vee a_{2} \vee \cdots \vee a_{n}$ has a Rad-supplement in L and $a_{i} / 0$ is cofinitely Radsupplemented for every $i=1,2, \ldots, n$, then $x$ has a Rad-supplement in $L$. 
Proof. Clear from Lemma 1.

Lemma 2. Let $L$ be a lattice, I be a nonempty index set and $a_{i} \in L$ for every $i \in I$. If $1=\underset{i \in I}{\vee} a_{i}$ and $a_{i} / 0$ is cofinitely Rad-supplemented for every $i \in I$, then $L$ is also cofinitely Rad-supplemented.

Proof. Let $a$ be any cofinite element of $L$. By hypothesis, $1=\underset{i \in I}{\vee} a_{i}=\underset{i \in I}{\vee}\left(a \vee a_{i}\right)$. Since $1 / a$ is compact and $a \vee a_{i} \in 1 / a$ for every $i \in I$, there exist $i_{1}, i_{2}, \ldots, i_{n} \in I$ such that $1=a \vee a_{i_{1}} \vee a_{i_{2}} \vee \ldots \vee a_{i_{n}}$. Since 0 is a Rad-supplement of $1=a \vee a_{i_{1}} \vee a_{i_{2}} \vee \ldots \vee a_{i_{n}}$ and $a_{i_{t}} / 0$ is cofinitely Rad-supplemented for every $t=1,2, \ldots, n$, by Corollary $1, a$ has a Rad-supplement in $L$. Hence $L$ is cofinitely Rad-supplemented.

Corollary 2. Let $L$ be a lattice and $1=a_{1} \vee a_{2} \vee \ldots \vee a_{n}$ in $L$. If $a_{i} / 0$ is cofinitely Rad-supplemented for every $i=1,2, \ldots, n$, then $L$ is also cofinitely Rad-supplemented.

Proof. Clear from Lemma 2.

Proposition 2. Let $L$ be a cofinitely Rad-supplemented lattice and $a \in L$. Then $1 / a$ is also cofinitely Rad-supplemented.

Proof. Let $x$ be any cofinite element of $1 / a$. Then $1 / x$ is compact and $x$ is a cofinite element of $L$. Since $L$ is cofinitely Rad-supplemented, $x$ has a Rad-supplement $y$ in $L$. Since $a \leq x$, by [3, Lemma 5], $a \vee y$ is a Rad-supplement of $x$ in $1 / a$. Hence $1 / a$ is cofinitely Rad-supplemented.

Proposition 3. Let $L$ be a cofinitely Rad-supplemented lattice. Then every cofinite element of $1 / r(L)$ is a direct summand of $1 / r(L)$.

Proof. Let $x$ be any cofinite element of $1 / r(L)$. Then $1 / x$ is compact and $x$ is a cofinite element of $L$. Since $L$ is cofinitely Rad-supplemented, $x$ has a Radsupplement $y$ in $L$. Here $1=x \vee y$ and $x \wedge y \leq r(y / 0) \leq r(L)$. Then $1=x \vee y \vee r(L)$ and since $r(L) \leq x, x \wedge(y \vee r(L))=(x \wedge y) \vee r(L)=r(L)$. Hence $1=x \oplus(y \vee r(L))$ in $1 / r(L)$ and $x$ is a direct summand of $1 / r(L)$.

\section{Cofinitely Weak Radical Supplemented Lattices}

In this part, cofinitely weak radical supplemented lattices are defined and some properties of them are given.

Definition 2. Let $L$ be a lattice and $a, b \in L$. If $a \vee b=1$ and $a \wedge b \leq r(L)$, then $b$ is called a weak radical supplement (or briefly, weak Rad-supplement) of $a$ in $L$.

Definition 3. Let $L$ be a lattice. If every element of $L$ has a weak Rad-supplement in $L$, then $L$ is called a weakly radical supplemented (or weakly Rad-supplemented) lattice. If every cofinite element of $L$ has a weak Rad-supplement in $L$, then $L$ is called a cofinitely weak radical supplemented (or cofinitely weak Rad-supplemented) lattice. 
It is clear that every cofinitely weak supplemented lattice is cofinitely weak Radsupplemented. It is also clear that every cofinitely Rad-supplemented lattice is cofinitely weak Rad-supplemented.

Proposition 4. Let $L$ be a cofinitely weak Rad-supplemented lattice. If $r(L) \ll L$, then $L$ is cofinitely weak supplemented.

Proof. Clear from definitions.

Proposition 5. Let $L$ be a compact lattice. Then $L$ is cofinitely weak Radsupplemented if and only if $L$ is weakly Rad-supplemented.

Proof. Clear, since every element of $L$ is cofinite.

Lemma 3. Let $L$ be a lattice, $a \in L$ and $x$ be a cofinite element of $L$. If $x \vee$ a has a weak Rad-supplement in L and a/0 is cofinitely weak Rad-supplemented, then $x$ has a weak Rad-supplement in $L$.

Proof. Let $b$ be a weak Rad-supplement of $x \vee a$ in $L$. Then $x \vee a \vee b=1$ and $(x \vee a) \wedge b \leq r(L)$. Since $x$ is a cofinite element of $L$, we clearly see that $x \vee b$ is a cofinite element of $L$. Then by $\frac{1}{x \vee b}=\frac{x \vee a \vee b}{x \vee b} \cong \frac{a}{a \wedge(x \vee b)}, a \wedge(x \vee b)$ is a cofinite element of $a / 0$. Since $a / 0$ is cofinitely weak Rad-supplemented, $a \wedge(x \vee b)$ has a weak Rad-supplement $c$ in $a / 0$. Here $(a \wedge(x \vee b)) \vee c=a$ and $c \wedge(x \vee b)=c \wedge a \wedge$ $(x \vee b) \leq r(a / 0) \leq r(L)$. Hence $1=x \vee a \vee b=x \vee b \vee(a \wedge(x \vee b)) \vee c=x \vee b \vee c$ and $x \wedge(b \vee c) \leq(b \wedge(x \vee c)) \vee(c \wedge(x \vee b)) \leq(b \wedge(x \vee a)) \vee r(L) \leq r(L) \vee r(L)=r(L)$. Thus $b \vee c$ is a weak Rad-supplement of $x$ in $L$.

Corollary 3. Let $L$ be a lattice, $a_{1}, a_{2}, \ldots, a_{n} \in L$ and $x$ be a cofinite element of $L$. If $x \vee a_{1} \vee a_{2} \vee \cdots \vee a_{n}$ has a weak Rad-supplement in $L$ and $a_{i} / 0$ is cofinitely weak Rad-supplemented for every $i=1,2, \ldots, n$, then $x$ has a weak Rad-supplement in $L$.

Proof. Clear from Lemma 3.

Lemma 4. Let $L$ be a lattice, I be a nonempty index set and $a_{i} \in L$ for every $i \in I$. If $1=\underset{i \in I}{\vee} a_{i}$ and $a_{i} / 0$ is cofinitely weak Rad-supplemented for every $i \in I$, then $L$ is also cofinitely weak Rad-supplemented.

Proof. Let $a$ be any cofinite element of $L$. By hypothesis, $1=\underset{i \in I}{\vee} a_{i}=\underset{i \in I}{\vee}\left(a \vee a_{i}\right)$. Since $1 / a$ is compact and $a \vee a_{i} \in 1 / a$ for every $i \in I$, there exist $i_{1}, i_{2}, \ldots, i_{n} \in I$ such that $1=a \vee a_{i_{1}} \vee a_{i_{2}} \vee \ldots \vee a_{i_{n}}$. Since 0 is a weak Rad-supplement of $1=a \vee a_{i_{1}} \vee$ $a_{i_{2}} \vee \ldots \vee a_{i_{n}}$ and $a_{i_{t}} / 0$ is cofinitely weak Rad-supplemented for every $t=1,2, \ldots, n$, by Corollary 3, $a$ has a weak Rad-supplement in $L$. Hence $L$ is cofinitely weak Radsupplemented.

Corollary 4. Let $L$ be a lattice and $1=a_{1} \vee a_{2} \vee \ldots \vee a_{n}$ in $L$. If $a_{i} / 0$ is cofinitely weak Rad-supplemented for every $i=1,2, \ldots, n$, then $L$ is also cofinitely weak Radsupplemented. 
Proof. Clear from Lemma 4.

Lemma 5. Let $L$ be a lattice, $a, b, x \in L$ and $x \leq a$. If $b$ is a weak Rad-supplement of $a$ in $L$, then $x \vee b$ is a weak Rad-supplement of $a$ in $1 / x$.

Proof. Since $b$ is a weak Rad-supplement of $a$ in $L, a \vee b=1$ and $a \wedge b \leq r(L)$. Then $a \vee x \vee b=1$ and $a \wedge(x \vee b) \leq x \vee(a \wedge b) \leq x \vee r(L) \leq r(1 / x)$. Hence $x \vee b$ is a weak Rad-supplement of $a$ in $1 / x$.

Proposition 6. Let $L$ be a cofinitely weak Rad-supplemented lattice and $a \in L$. Then $1 /$ a is also cofinitely weak Rad-supplemented.

Proof. Let $x$ be any cofinite element of $1 / a$. Then $1 / x$ is compact and $x$ is a cofinite element of $L$. Since $L$ is cofinitely weak Rad-supplemented, $x$ has a weak Rad-supplement $y$ in $L$. Since $a \leq x$, by Lemma 5, $a \vee y$ is a weak Rad-supplement of $x$ in $1 / a$. Hence $1 / a$ is cofinitely weak Rad-supplemented.

Proposition 7. Let $L$ be a lattice. Then $L$ is cofinitely weak Rad-supplemented if and only if every cofinite element of $1 / r(L)$ is a direct summand of $1 / r(L)$.

Proof. $(\Longrightarrow)$ Let $x$ be any cofinite element of $1 / r(L)$. Then $1 / x$ is compact and $x$ is a cofinite element of $L$. Since $L$ is cofinitely weak Rad-supplemented, $x$ has a weak Rad-supplement $y$ in $L$. Here $1=x \vee y$ and $x \wedge y \leq r(L)$. Then $1=x \vee y \vee r(L)$ and since $r(L) \leq x, x \wedge(y \vee r(L))=(x \wedge y) \vee r(L)=r(L)$. Hence $1=x \oplus(y \vee r(L))$ in $1 / r(L)$ and $x$ is a direct summand of $1 / r(L)$.

$(\Longleftarrow)$ Let $x$ be any cofinite element of $L$. Here clearly we can see that $x \vee r(L)$ is a cofinite element of $1 / r(L)$. By hypothesis, $x \vee r(L)$ is a direct summand of $1 / r(L)$. Then there exists $y \in 1 / r(L)$ such that $1=x \vee r(L) \vee y=x \vee y$ and $(x \vee r(L)) \wedge y=$ $r(L)$. Since $r(L) \leq y$, by modularity, $r(L)=(x \vee r(L)) \wedge y=(x \wedge y) \vee r(L)$ and $x \wedge y \leq r(L)$. Hence $y$ is a weak Rad-supplement of $x$ in $L$. Therefore, $L$ is cofinitely weak Rad-supplemented.

Proposition 8. Let $L$ be a lattice and $a \ll L$. If $1 / a$ is cofinitely weak Radsupplemented, then $L$ is also cofinitely weak Rad-supplemented.

Proof. Let $x$ be any cofinite element of $L$. Clearly we see that $x \vee a$ is a cofinite element of $1 / a$. Since $1 / a$ is cofinitely weak Rad-supplemented, $x \vee a$ has a weak $\operatorname{Rad}$-supplement $y$ in $1 / a$. Here $x \vee a \vee y=1$ and $(x \vee a) \wedge y \leq r(1 / a)$. Since $x \vee$ $a \vee y=1$ and $a \leq y, x \vee y=1$. Since $a \ll L$, clearly we see that $r(1 / a)=r(L)$. Hence $x \vee y=1$ and $x \wedge y \leq(x \vee a) \wedge y \leq r(1 / a)=r(L)$. Thus $L$ is cofinitely weak Rad-supplemented.

Let $x, y \in L$. It is defined a relation $\beta_{*}$ on the elements of $L$ by $x \beta_{*} y$ if and only if for every $t \in L$ with $x \vee t=1$ then $y \vee t=1$ and for every $k \in L$ with $y \vee k=1$ then $x \vee k=1$. The definition of $\beta_{*}$ relation and some properties of this relation are in [6]. The definition of $\beta^{*}$ relation on modules and some properties of this relation are in [4]. 
Lemma 6. Let $L$ be a lattice. If every cofinite element of $L$ is $\beta_{*}$ equivalent to a weak Rad-supplement element in $L$, then $L$ is cofinitely weak Rad-supplemented.

Proof. Let $x$ be a cofinite element of $L$. By hypothesis, there exists a weak Radsupplement element $y$ in $L$ such that $x \beta_{*} y$. Let $y$ is a weak Rad-supplement of $a$ in $L$. Here $y \vee a=1$ and $y \wedge a \leq r(L)$. Since $x \beta_{*} y$ and $y \vee a=1, x \vee a=1$. Assume $x \wedge a \not \leq r(L)$. Then there exists a maximal $(\neq 1)$ element $t$ of $L$ with $x \wedge a \not \leq t$. Here $(x \wedge a) \vee t=1$. By [6, Lemma 2], $x \vee(a \wedge t)=1$ and since $x \beta_{*} y, y \vee(a \wedge t)=1$. Since $a \vee t=1$, by [6, Lemma 2], $(y \wedge a) \vee t=1$. Since $y \wedge a \leq r(L) \leq t, t=(y \wedge a) \vee t=$ 1. This contradicts with $t \neq 1$. Hence $x \wedge a \leq r(L)$. Therefore, $a$ is a weak Radsupplement of $x$ in $L$ and $L$ is cofinitely weak Rad-supplemented.

Corollary 5. Let $L$ be a lattice. If every cofinite element of $L$ lies above a weak Rad-supplement element in $L$, then $L$ is cofinitely weak Rad-supplemented.

Proof. Clear from Lemma 6.

\section{REFERENCES}

[1] R. Alizade and S. E. Toksoy, "Cofinitely weak supplemented lattices," Indian J. Pure Appl. Math., vol. 40, no. 5, pp. 337-346, 2009.

[2] R. Alizade and S. E. Toksoy, "Cofinitely supplemented modular lattices," Arab. J. Sci. Eng., vol. 36, no. 6, pp. 919-923, 2011, doi: 10.1007/s13369-011-0095-z. [Online]. Available: https://doi.org/10.1007/s13369-011-0095-z

[3] Ç. Biçer, C. Nebiyev, and A. Pancar, "Generalized supplemented lattices," Miskolc Math. Notes, vol. 19, no. 1, pp. 141-147, 2018, doi: 10.18514/mmn.2018.1974. [Online]. Available: https://doi.org/10.18514/mmn.2018.1974

[4] G. F. Birkenmeier, F. Takil Mutlu, C. Nebiyev, N. Sokmez, and A. Tercan, "Goldie*-supplemented modules," Glasg. Math. J., vol. 52, no. A, pp. 41-52, 2010, doi: 10.1017/S0017089510000212. [Online]. Available: https://doi.org/10.1017/S0017089510000212

[5] G. Călugăreanu, Lattice concepts of module theory, ser. Kluwer Texts in the Mathematical Sciences. Kluwer Academic Publishers, Dordrecht, 2000, vol. 22. [Online]. Available: https://doi.org/10.1007/978-94-015-9588-9. doi: 10.1007/978-94-015-9588-9

[6] C. Nebiyev and H. H. Ökten, " $\beta_{*}$ relation on lattices," Miskolc Math. Notes, vol. 18, no. 2, pp. 993-999, 2017, doi: 10.18514/mmn.2017.1782. [Online]. Available: https: //doi.org/10.18514/mmn.2017.1782

[7] E. Türkmen and A. Pancar, "On cofinitely Rad-supplemented modules," Int. J. Pure Appl. Math., vol. 53, no. 2, pp. 153-162, 2009.

[8] Y. Wang and N. Ding, "Generalized supplemented modules," Taiwanese J. Math., vol. 10, no. 6, pp. 1589-1601, 2006, doi: 10.11650/twjm/1500404577. [Online]. Available: https://doi.org/10.11650/twjm/1500404577

[9] R. Wisbauer, Foundations of module and ring theory, ser. Algebra, Logic and Applications. Gordon and Breach Science Publishers, Philadelphia, PA, 1991, vol. 3, a handbook for study and research.

[10] H. Zöschinger, "Komplementierte Moduln über Dedekindringen,” J. Algebra, vol. 29, pp. 42-56, 1974, doi: 10.1016/0021-8693(74)90109-4. [Online]. Available: https://doi.org/10.1016/ 0021-8693(74)90109-4 
Authors' addresses

\section{Celıl Nebiyev}

Ondokuz Mayıs University, Department of Mathematics, Kurupelit-Atakum, Samsun, Turkey

E-mail address: cnebi yev@omu.edu.tr

Hasan Hüseyın Ökten

Amasya University, Technical Sciences Vocational School, Amasya, Turkey

E-mail address: hokten@gmail.com 